\title{
Proper motion analysis of the jet of R Aquarii
}

\author{
K. Mäkinen ${ }^{1}$, H. J. Lehto ${ }^{1,2}$, R. Vainio ${ }^{3}$, and D. R. H. Johnson ${ }^{4}$ \\ 1 Tuorla Observatory, Väisäläntie 20, 21500 Piikkiö, Finland \\ e-mail: krikokko@utu.fi \\ 2 Department of Physics, 20014 Turku University, Finland \\ 3 Department of Physical Sciences, PO Box 64, 00014 University of Helsinki, Finland \\ ${ }^{4}$ Charterhouse, Godalming, Surrey, GU7 2DX, UK
}

Received 15 December 2003 / Accepted 18 May 2004

\begin{abstract}
We have observed the jet of R Aquarii at high resolution with the VLA in 1992.83 and in 1999.78. Observations in the first epoch have resolved the base of the jet which shows a helical structure. We cannot detect the expected new jet component at either epoch. This does not disprove the idea of periodic ejection. Either the timing inferred from the acceleration models, or the assumed periastron passage is incorrect. Alternatively, a single new component cannot be resolved due to the dense core. Proper motion analysis of the jet components shows that previously derived acceleration models do not fit our new data. Indeed, the first $\sim 1^{\prime \prime}$ of the jet, both to north-east and south-west, appears fixed and has slowly moving shocks at the termination points, whereas the positions of the outer components fit best a ballistic orbit. We propose that the components are formed due to enhanced matter flow at periastron, accelerated during the first $1^{\prime \prime}$ and then ejected as bullets. Component A at a distance of $\sim 4^{\prime \prime}$ from the core has broken into two parts, similar to what was previously assumed to have happened to the outermost components $\mathrm{B}$ and $\mathrm{D}$. The disruption is probably a consequence of a reverse shock or a collision with a cloud.
\end{abstract}

Key words. stars: binaries: symbiotic - stars: individual: R Aqr - ISM: jets and outflows

\section{Introduction}

$\mathrm{R}$ Aqr is a dusty-type (D) symbiotic binary with a mass-losing $\sim 1.75 M_{\odot}$ M7III (Lang 1992) Mira-type long-period variable (LPV) as the primary star. The $\sim 1.0 M_{\odot}$ hot companion is assumed to be a white dwarf and it is presumably surrounded by an accretion disk, which gives rise to a jet (Kafatos \& Michalitsianos 1982). The jet is composed of some discrete features although it shows continuous characteristics as well. It extends up to $10^{\prime \prime}$ (=2000 AU) north-east (NE) of the core. The fainter counter-jet to the SW is detectable with the VLA only up to a distance of several arc-seconds, but in X-ray, features have been found even $30^{\prime \prime}$ from the core (Kellogg et al. 2001). The radio features are called as $\mathrm{A}^{\prime}, \mathrm{C} 1$ (core), C2, A, B and D from south-west (SW) to NE (see Fig. 1). The best-observed jet component is feature A covering almost 20 years in time. The jet is also seen in the UV (first by Michalitsianos \& Kafatos 1982). Both the binary system and the jet are embedded in a complex nebula (150" EW and 80" NS; Solf \& Ulrich 1985; Hollis et al. 1999), which is best seen in the optical but can be detected at radio wavelengths as well (Hollis et al. 1987). The distance to R Aqr is 197 pc (Van Leeuwen 1997).

The production of the jet features has been explained in several ways. Some of the mechanisms proposed have been based on stellar wind or collision of winds models (e.g., Burgarella et al. 1992). Particularly in recent studies, interaction of binary components has been thought to be relevant to the jet formation mechanism and most analyses have utilized the compact companion/accretion disk scenario (e.g., Kellogg et al. 2001; Hollis \& Koupelis 2000; Meier \& Kafatos 1995; Solf 1992; Lehto \& Johnson 1992, LJ hereafter; Sopka et al. 1982). In these scenarios the matter flowing towards the hot companion is most likely enhanced during periaston passage. Also Roche lobe overflow can be possible in an orbit with $a \sim 2.6 \times 10^{14} \mathrm{~cm}$ (e.g., Hollis et al. 1997b), if the orbit is highly elliptical. There are no direct observations of the secondary, but in radio frequency, Hollis et al. (1997c) have simultaneously observed the continuum emission and the $\mathrm{SiO}$ maser emission from the core region in $43 \mathrm{GHz}$. They have resolved two components which they suggest represent the two stars of the binary. The offset of these components was $55 \pm 2$ mas corresponding to $\sim 11 \mathrm{AU}$. In addition, Kellogg et al. (2001) detected a $6.4 \mathrm{keV}$ spike in their X-ray observations, which was interpreted as due to an unresolved hard source, probably hidden from the view by an edge-on accretion disk.

The visible light curve of $\mathrm{R}$ Aqr suggests an activity modulation of 44 years (Willson et al. 1981). This has been thought to indicate the binary period. Based on the features in optical spectra (Willson et al. 1981) it has been claimed that we see the system nearly edge-on and that reduced activity levels, perhaps because of eclipses, in the Mira variability took place in 1890, 1934 and 1978. Merrill (1950) showed that the blue 


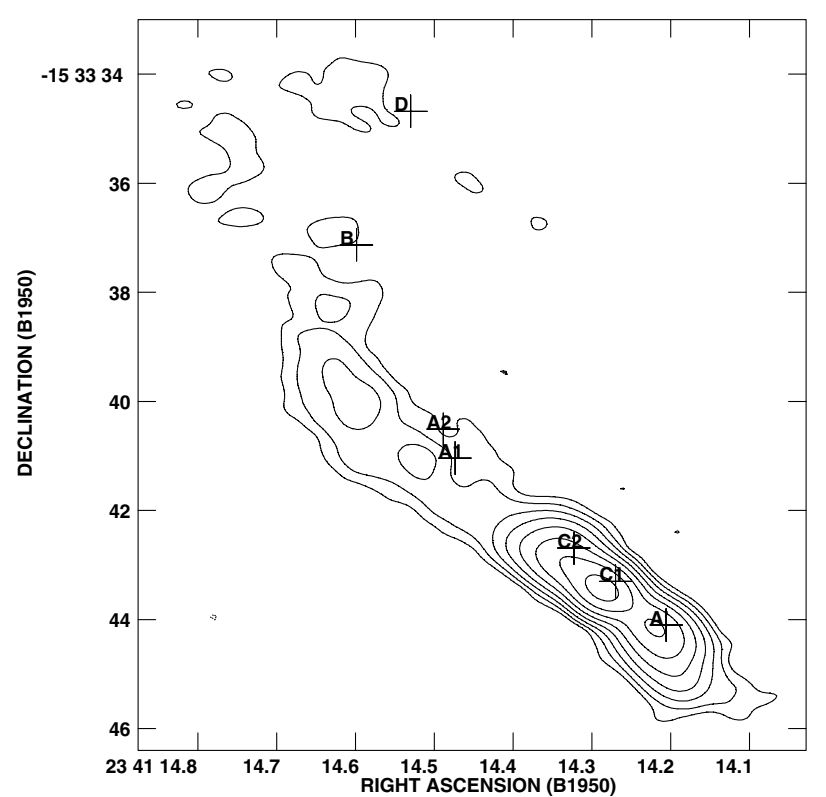

Fig. 1. The R Aqr jet in $8 \mathrm{GHz}$ observed in 1999.78. The peak flux is 10.4 mJy. Contour levels are $-0.06,0.06,0.12,0.24,0.48,0.96,1.92$, $3.84,7.68 \mathrm{mJy}$. The beam size is $780 \times 540 \mathrm{mas}\left(\mathrm{PA} 73^{\circ}\right)$ and the rms noise is $17 \mu \mathrm{Jy}$. Lower contours are used here to show the outer low flux density areas. The crosses show the position of each component in 1992.83, except B and D in 1991.63 (LJ).

companion had little effect on the spectrum of the LPV during 1934-1949, which indicated that the companion was moving behind the LPV in its orbit. Further, no trace of the hot companion in the spectroscopic plates was seen in the 1965 Mira minimum (Ilovaisky \& Spinrad 1966). The duration of the eclipsing event between 1974 and 1980 indicated that the occulting companion was at apastron. In this scenario, periastron passage would therefore occur at an orbital phase opposite to the eclipse i.e. around year 2000, assuming the claimed 44-year period. However, there is another point of view proposed by Hinkle et al. (1989) who pointed out that the white dwarf at apastron cannot eclipse the Mira on its own, because it is a much smaller body. But if the system is near periastron, the enhanced matter flow from the Mira filling its Roche lobe would illuminate the jet. The eclipsing body would be an extended thick accretion disk, which is cool in its outer regions. According to this explanation, the secondary would recently have been at apastron.

LJ observed that five jet components detectable at 8 and $5 \mathrm{GHz}$ had moved from their previous positions 6-10 years earlier. The results of proper motion analysis led to two possible models where the components were thought to be ejected from the central binary at periastron. In the first model (LJ1) the components were ejected as "bullets" with about a 20year interval. In the second model (LJ2) the jet components were linearly accelerated, possibly by a magnetic field, and ejected with about a $\sim 40$ yrs interval. According to the LJ1 model the components are on ballistic orbits, whereas in the LJ2 model the components should follow each other's tracks in a (twisted) magnetic field. The event separation time of the LJ2 model has been substantiated with HST observations (Hollis et al. 1997a), but models in the UV-region yielded nonlinear

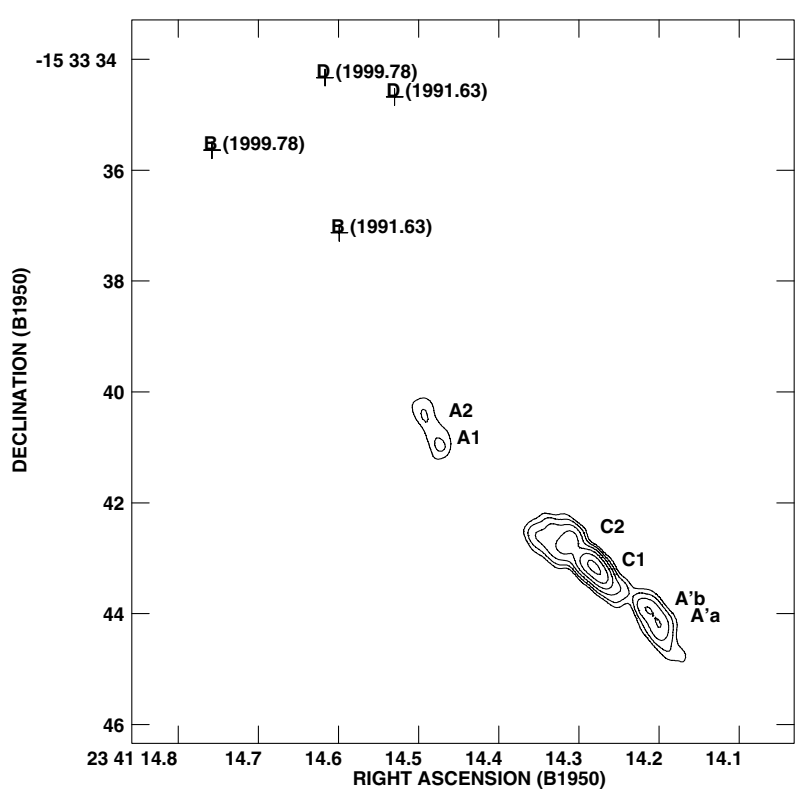

Fig. 2. The R Aqr jet in $8 \mathrm{GHz}$ observed in 1992.83. The peak flux is $6.4 \mathrm{mJy}$. Contour levels are $-0.17,0.17,0.33,0.66,1.32,2.64$ and $5.28 \mathrm{mJy}$. The beam size in this uniformly weighted map is $359 \times$ 228 mas (PA $\left.31^{\circ}\right)$. Note the two parts in A (NE) and $A^{\prime}(\mathrm{SW})$, the shock front in $\mathrm{C} 2$ and the $\mathrm{SW}$ extension in the core. The crosses show the positions of B and D in 1991.63 and in 1999.78.

acceleration. Characteristics of a helical structure were also seen. Component $\mathrm{A}^{\prime}$ was not included in these analyses since it appeared stationary and did not seem to fit to the laws.

Both models of LJ predicted that a new component would emerge from the core around year 1997. This corresponds to the next periastron passage of Willson et al. (1981). We were motivated to detect the new component. Two observation epochs, 1992.83 and 1999.78, also enabled us to compare the situations in the core region before and after the assumed ejection. In addition, our aim was to compare the positions of the radio components to the previously published data. In the present paper we present the analysis from the VLA data and compare it to $\mathrm{LJ}$, thus covering four observation epochs.

\section{Observations and data reduction}

\subsection{VLA 1992 observations}

Observations were made with the $\mathrm{NRAO}^{1}$ Very Large Array in 1992 October 30 in its "A" configuration. Measurements were made in 8 and $22 \mathrm{GHz}$, corresponding to $X$ and $K$ bands. All 27 antennae were available, utilizing an IF bandwidth of $50 \mathrm{MHz}$ and two IF pairs separated by $50 \mathrm{MHz}$.

At $8 \mathrm{GHz} \mathrm{R}$ Aqr was observed for $52 \mathrm{~min}$, in three $\sim 17$ min periods. $0316+413$ (3C 84) and $0134+329$ (3C 48) were used to calibrate the flux. 2345-167 was used as a phase calibrator.

1 The National Radio Astronomy Observatory is a facility of the National Science Foundation, operated under cooperative agreement by Associated Universities, Inc. 
The data obtained at $22 \mathrm{GHz}$ were more complicated to calibrate and therefore several calibration sources were used. $0316+413$ was used as a primary calibrator and the flux level was checked with $2201+315,2145+067$ and $2251+158$. These three additional sources have regularly been observed with the Metsähovi radio telescope at $22 \mathrm{GHz}$ (see Teräsranta et al. 1997). The phase calibrator was $2345-167$. The R Aqr onsource time was $3.8 \mathrm{~h}$.

\subsection{VLA 1999 observations}

These data were obtained with the VLA in October 12 and 13 1999 in its "BnA" configuration. Measurements were made at frequencies of 8,15 and $22 \mathrm{GHz}$ corresponding to $X, U$ and $K$ bands. All 27 antennae were used with the maximal bandwidth of $50 \mathrm{MHz}$ for the two polarizations in each IF pair. The $15 \mathrm{GHz}$ data will be considered in a separate paper.

The primary flux calibrator was 3C48 at all frequencies. At $22 \mathrm{GHz}$ fast switching phase calibration with a switching time of $3 \mathrm{~min} 47 \mathrm{~s}$ was used to reduce phase variations caused by the troposphere. The total on-source observing times were 7.5, 2.4 and 1.5 hours at 22,15 and $8 \mathrm{GHz}$, respectively. $2345-167$ was used as the phase calibrator at all frequencies.

\subsection{The maps and component fittings}

All the 1992 and 1999 data were calibrated and self-calibrated with the NRAO AIPS software package in the standard way. The coordinates of jet components were obtained by using the IMFIT and JMFIT tasks. All maps were $1024 \times 1024$ pixels with the cell size of $0{ }^{\prime} 05 \times 00^{\prime} 05$. Generally, components near the core were fitted using uniformly weighted maps or $22 \mathrm{GHz}$ maps so as not to hide the morphology under a too large beam. Outer jet components were fitted using natural weighted maps.

\subsubsection{The 1992 data}

The achieved rms noise level in the uniformly weighted $8 \mathrm{GHz}$ map (Fig. 2) is $35 \mu \mathrm{Jy}$, the theoretical noise being at the $20 \mu \mathrm{Jy}$ level. The beam size is $359 \times 228 \mathrm{mas}\left(\mathrm{PA} 31^{\circ}\right)$ and it was tapered with a Gaussian taper of $(215 \times 650) \mathrm{k} \lambda$ to match the $19998 \mathrm{GHz}$ beam (see next subsection). The untapered map revealed that the counter-jet component $\mathrm{A}^{\prime}$ has two parts, similar to Dougherty et al. (1995). In addition, the NE component A showed signs of breaking up into two parts. Gaussians were fitted to these sub-components ( $\mathrm{A}^{\prime} \mathrm{a}, \mathrm{A}^{\prime} \mathrm{b}, \mathrm{A} 1$ and $\mathrm{A} 2$ ) using the untapered map. The average position of $\mathrm{A}^{\prime}$ as well as components $\mathrm{C} 1$ and $\mathrm{C} 2$ were fitted using the tapered map (not shown). We also created a naturally weighted map with a convolving beam size equal to the $8 \mathrm{GHz}$ map of 1999. A Gaussian was then fitted to the A component on this map.

At $22 \mathrm{GHz}$ the theoretical rms noise with $3.8 \mathrm{~h}$ integration time would be $68 \mu \mathrm{Jy}$, but only a level of $100 \mu \mathrm{Jy}$ was achieved. The beam size in the natural weighted map (Fig. 3a) is $159 \times 121$ mas (PA 1.3). This beam was tapered with a Gaussian taper of $(550 \times 1000) \mathrm{k} \lambda$ to compare with the 1999 $22 \mathrm{GHz}$ data. The untapered $22 \mathrm{GHz}$ map resolved the core region (see Fig. 3a). Components $\mathrm{C} 1 \mathrm{c}$ (core), $\mathrm{C} 1 \mathrm{~b}$ and $\mathrm{Cla}$ according to Dougherty et al. (1995) were identified. As a result of the tapering, $\mathrm{Cla}$ and $\mathrm{C} 1 \mathrm{~b}$ are enclosed in $\mathrm{C} 1 \mathrm{a}+\mathrm{b}$ and positions of $\mathrm{Clc}$ and $\mathrm{Cla}+\mathrm{b}$ were fitted using the tapered map.

\subsubsection{The 1999 data}

The beam in the uniformly weighted map at $8 \mathrm{GHz}$ (not shown) is $673 \times 372\left(\mathrm{PA} 76^{\circ}\right)$ and the noise level is $19 \mu \mathrm{Jy}$. Gaussians were fitted to the components $\mathrm{A}^{\prime}$ (average position), $\mathrm{C} 1, \mathrm{C} 2$ on this map. We also made a natural weighted map (Fig. 1) to bring out the outermost components. This map has a noise level of $17 \mu \mathrm{Jy}$, which is very close to the theoretically expected thermal noise of $15 \mu \mathrm{Jy}$. The beam size is $785 \times 540$ mas $\left(\mathrm{PA} 73^{\circ}\right)$. This map was used to fit the positions of components A1, A2, B and D.

The $22 \mathrm{GHz}$ map (Fig. 3b) is uniformly weighted and has a beam size of $247 \times 151$ mas $\left(\mathrm{PA} 82^{\circ}\right.$ ). The rms noise level is $59 \mu \mathrm{Jy}$, close to the theoretically expected value of $46 \mu \mathrm{Jy}$. Positions of components $\mathrm{A}^{\prime} \mathrm{a}, \mathrm{A}^{\prime} \mathrm{b}, \mathrm{Clc}$ (core) and $\mathrm{C} 1 \mathrm{a}+\mathrm{b}$ were fitted using this map.

\section{Results and discussion}

To compare the positions of components relative the core in all four epochs (those reported in LJ and two from here), we first aimed to choose the component $\mathrm{C} 1$ of $8 \mathrm{GHz}$ as a reference of the core (similarly to LJ), but it turned out that the proper motion speed of the core varied considerably. This could be caused by the binary motion but is probably due to brightness variations. The tapered $199222 \mathrm{GHz}$ map reveals that the integral intensity of $\mathrm{C} 1 \mathrm{a}+\mathrm{b}$ is $\sim 80 \%$ of the core's intensity, but in 1999 it is only $40 \%$. This obviously lifts the position of $\mathrm{C} 1$ in the $19928 \mathrm{GHz}$ data and probably also in 1991. Cla+b was also noted as the brightest component in the study of Dougherty et al. (1995). For this reason we adopted the core position from the $22 \mathrm{GHz}$ map. The proper motion of the $22 \mathrm{GHz}$ core, $\left(00^{\prime} 024,-0.031\right) \mathrm{yr}^{-1}$, is also more similar to the optical proper motion, e.g. (0.'0297, $-0{ }^{\prime}$ '0328) $\mathrm{yr}^{-1}$ (Kharchenko et al. 2002), than that in $8 \mathrm{GHz}$, which has measured to be $\left(0{ }^{\prime} 008,-0 \prime^{\prime} 040\right) \mathrm{yr}^{-1}$ between 1992.83 and 1999.78.

The positions of all visible components in the two epochs are listed in Table 1. The proper motion velocities are given in Table 2, where the analysis of the core region is also shown. Figure 6 shows the positions in all four epochs.

\subsection{Magnetic base in the jet?}

Comparison of Figs. $3 \mathrm{a}$ and $3 \mathrm{~b}$ reveals that no new components are seen to emerge from the core between 1992.83 and 1999.78 within the resolution. This non-detection does not rule out the idea of periodic ejection. First, according to Hinkle et al. (1989), the companion may just have been at apastron and no emergence is expected. This is inconsistent with the ejection times predicted by the LJ models. Second, the timing could be just slightly wrong and $\mathrm{C} 1 \mathrm{a}+\mathrm{b}$ actually represents a new component, which was already formed around 1990. As was mentioned above, the relative brightness of $\mathrm{C} 1 \mathrm{a}+\mathrm{b}$ was high around 

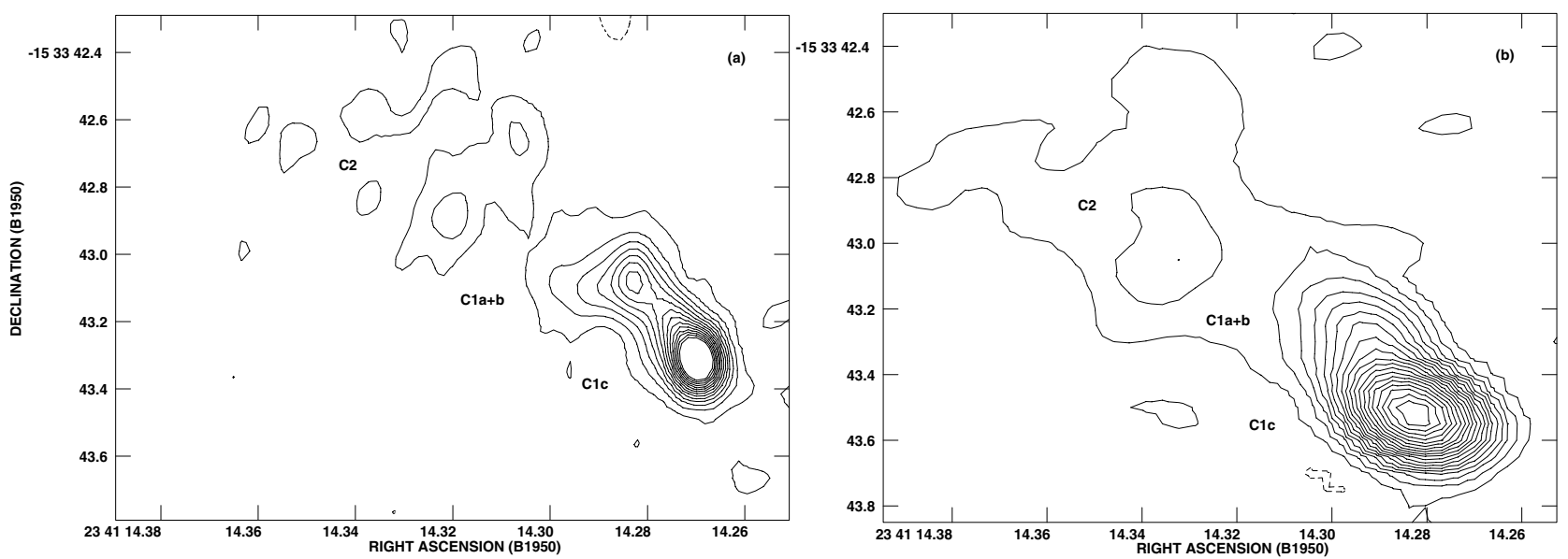

Fig. 3. The R Aqr jet in $22 \mathrm{GHz}$ in 1992.83 a) and in 1999.78 b) showing the core region. Note that both maps are untapered. No new component can be seen emerging from the core unless it had already emerged before 1992 (see discussion in text). a) The peak flux is $7.37 \times 10^{-3} \mathrm{Jy}$. Contours drawn are $-0.25,0.25,0.60,0.96,1.31,1.66,2.02,2.37,2.73,3.08,3.43,3.79,4.14,4.49,4.85$ and 5.20 mJy. The beam size in this uniform weighted map is $159 \times 121$ mas (PA 1:3) and the rms noise is $100 \mu \mathrm{Jy}$. The integral intensities of C1c, C1b and C1a are 10.11, 7.32 and 2.39 mJy, respectively. b) The peak flux is $1.19 \times 10^{-2} \mathrm{Jy}$. Contours drawn are $-0.14,0.14,0.37,0.63,0.91,1.35,1.22,1.56,1.93,2.34$, $2.79,3.29,3.83,4.46,5.10,5.82,6.62,7.50,8.47,9.54$ and $10.71 \mathrm{mJy}$. The beam size in this uniform weighted map is $247 \times 151 \mathrm{mas}\left(\mathrm{PA} 82^{\circ}\right)$ and the rms noise is $59 \mu \mathrm{Jy}$. The integral intensities of $\mathrm{C} 1 \mathrm{a}+\mathrm{b}$ and $\mathrm{C} 1 \mathrm{c}$ are $6.61 \mathrm{mJy}$ and $14.73 \mathrm{mJy}$, respectively.

Table 1. RA, Dec and deconvolved position angle of the components from AIPS IMFIT solution in 1992.83 and 1999.78 are listed in Cols. $2-4$. Column 5 gives the angle of the position vector of the component relative to positive Dec-axis (an average value). Column 6 lists the distances from the core.

\begin{tabular}{|c|c|c|c|c|c|}
\hline (1) & $\begin{array}{r}\mathrm{RA} \\
(\mathrm{h} \text { min s) } \\
(2)\end{array}$ & $\begin{array}{r}\text { Dec } \\
\left({ }^{\prime \prime}\right) \\
(3)\end{array}$ & $\begin{array}{c}\text { PA of } \\
\text { feature (deg) } \\
\text { (4) }\end{array}$ & $\begin{array}{c}\text { PA of position } \\
\text { vector (deg) } \\
\text { (5) }\end{array}$ & $\begin{array}{c}\text { Distance } \\
\text { from C1c (") } \\
(6)\end{array}$ \\
\hline \multicolumn{6}{|l|}{1992.83} \\
\hline $\mathrm{A}^{\prime}$ & 234114.208 & -153344.07 & 38 & 230 & 1.18 \\
\hline $\mathrm{A}^{\prime} \mathrm{a}$ & 14.202 & 44.17 & 22 & 229 & 1.31 \\
\hline$A^{\prime} b$ & 14.219 & 43.87 & 62 & 233 & 0.92 \\
\hline $\mathrm{C} 1$ (8 GHz) & 14.281 & 43.18 & 47 & 50 & 0.20 \\
\hline $\mathrm{C} 1 \mathrm{c}(22 \mathrm{GHz})$ & 14.270 & 43.31 & 55 & - & 0.00 \\
\hline $\mathrm{C} 1 \mathrm{a}+\mathrm{b}$ & 14.283 & 43.10 & 74 & 41 & 0.28 \\
\hline $\mathrm{C} 2$ & 14.325 & 42.66 & 81 & 51 & 1.02 \\
\hline A & 14.482 & 40.67 & 31 & 49 & 4.03 \\
\hline A1 & 14.474 & 41.04 & 148 & 52 & 3.70 \\
\hline $\mathrm{A} 2$ & 14.489 & 40.51 & 26 & 48 & 4.21 \\
\hline \multicolumn{6}{|l|}{1999.78} \\
\hline $\mathrm{A}^{\prime}$ & 14.218 & 44.21 & 37 & 233 & 1.15 \\
\hline $\mathrm{A}^{\prime} \mathrm{a}$ & 14.204 & 44.47 & 140 & 230 & 1.47 \\
\hline $\mathrm{A}^{\prime} \mathrm{b}$ & 14.223 & 44.09 & 149 & 236 & 1.02 \\
\hline $\mathrm{C} 1(8 \mathrm{GHz})$ & 14.285 & 43.46 & 32 & 34 & 0.08 \\
\hline $\mathrm{C} 1 \mathrm{c}(22 \mathrm{GHz})$ & 14.282 & 43.52 & 43 & - & 0.00 \\
\hline $\mathrm{C} 1 \mathrm{a}+\mathrm{b}$ & 14.291 & 43.35 & 23 & 37 & 0.21 \\
\hline $\mathrm{C} 2$ & 14.330 & 42.96 & 59 & 51 & 0.88 \\
\hline A1 & 14.524 & 41.11 & 59 & 55 & 4.23 \\
\hline $\mathrm{A} 2$ & 14.605 & 39.77 & 67 & 51 & 5.97 \\
\hline B & 14.758 & 35.64 & 8 & 41 & 10.44 \\
\hline D & 14.617 & 34.33 & 92 & 28 & 10.37 \\
\hline
\end{tabular}

1992. However, $\mathrm{C} 1 \mathrm{a}+\mathrm{b}$ does not seem to move away from the core indicating that it is a part of a fixed base of the jet. Third, the core could be very dense and a new component cannot be resolved. Fourth, the emerging component could first proceed very quickly up to a shock front (probably $\mathrm{C} 2$, see the explanation below), collide with it and thus almost stop and then travel on a ballistic orbit. This means that a new component could leave much later than in the $\mathrm{LJ} 2$ model. If the temporary 
Table 2. Absolute velocities of the components are listed in Cols. 2-4. Proper motions and position angles of the components relative the core between 1992.83 and 1999.78 (except components B and D between 1991.63-1999.78) are listed in Cols. 6 and 7. Last two Cols. (8 and 9) represents the parallel and perpendicular components of the proper motions relative the core. The parallel component $\mu_{\|}$indicates the vector component between the direction of $\mu$ and the position angle of the position vector relative the core as an average between the two epochs. The perpendicular component $\mu_{\perp}$ is orthogonal to that.

\begin{tabular}{|c|c|c|c|c|c|c|c|c|}
\hline \multirow[b]{2}{*}{ Component } & \multicolumn{4}{|c|}{ Absolute motion } & \multicolumn{4}{|c|}{ Motion relative to core } \\
\hline & $\begin{array}{r}\Delta \mathrm{RA} \\
\left({ }^{\prime \prime} \mathrm{yr}^{-1}\right) \\
(2)\end{array}$ & $\begin{array}{r}\Delta \text { Dec } \\
\left({ }^{\prime \prime} \mathrm{yr}^{-1}\right) \\
(3)\end{array}$ & $\begin{array}{c}\text { Absolute } \\
\left({ }^{\prime \prime} \mathrm{yr}^{-1}\right) \\
(4)\end{array}$ & $\begin{array}{c}\text { PA for } \\
\text { absolute (deg) } \\
\text { (5) }\end{array}$ & $\begin{array}{c}\mu \text { relative } \\
\text { to } \mathrm{C} 1\left({ }^{\prime \prime} \mathrm{yr}^{-1}\right) \\
\text { (6) }\end{array}$ & $\begin{array}{l}\text { PA for } \\
\mu \text { (deg) } \\
\quad(7)\end{array}$ & $\begin{array}{r}\mu_{\|} \\
\left(" \mathrm{yr}^{-1}\right) \\
(8)\end{array}$ & $\begin{array}{r}\mu_{\perp} \\
\left(\mathrm{yr}^{-1}\right) \\
(9)\end{array}$ \\
\hline $1992.83-1999.78$ & & & & & & & & \\
\hline $\mathrm{A}^{\prime}$ & 0.0209 & -0.019 & 0.029 & 133 & 0.012 & -17 & -0.004 & 0.011 \\
\hline $\mathrm{A}^{\prime} \mathrm{a}$ & 0.0050 & -0.044 & 0.044 & 174 & 0.023 & 236 & -0.023 & -0.003 \\
\hline $\mathrm{A}^{\prime} \mathrm{b}$ & 0.0085 & -0.032 & 0.033 & 165 & 0.016 & 264 & -0.014 & -0.008 \\
\hline C1 (8 GHz) & 0.0082 & -0.040 & 0.041 & 169 & 0.019 & 239 & -0.018 & -0.005 \\
\hline $\mathrm{C} 1 \mathrm{c}(22 \mathrm{GHz})$ & 0.0240 & -0.031 & 0.039 & 142 & 0.000 & - & 0.000 & 0.000 \\
\hline $\mathrm{C} 1 \mathrm{a}+\mathrm{b}$ & 0.0166 & -0.036 & 0.039 & 155 & 0.009 & 237 & -0.009 & -0.003 \\
\hline $\mathrm{C} 2$ & 0.0101 & -0.043 & 0.044 & 167 & 0.019 & 229 & -0.019 & -0.001 \\
\hline A1 & 0.1035 & -0.011 & 0.104 & 96 & 0.082 & 76 & 0.076 & 0.031 \\
\hline A2 & 0.2403 & 0.107 & 0.263 & 66 & 0.256 & 57 & 0.254 & 0.035 \\
\hline B & 0.2800 & 0.183 & 0.335 & 57 & 0.333 & 50 & 0.327 & 0.063 \\
\hline D & 0.1527 & 0.043 & 0.159 & 74 & 0.148 & 60 & 0.121 & 0.085 \\
\hline
\end{tabular}

change in the position of the $8 \mathrm{GHz}$ core is caused by brightness variations due to flowing gas, the gas would have most probably been emitted during the previous periastron passage, probably near 1978 according to Hinkle et al. (1989).

The morphology of the NE component C2 shows features of a transverse planar shock both in $22 \mathrm{GHz}$ and $8 \mathrm{GHz}$ (cf. Figs. 2 and 3a), especially in 1992. This structure as well as the low intensity gas trailing right after $\mathrm{C} 2$ are similar to the structures observed with HST (Paresce \& Hack 1994; Hollis et al. 1997a). These features could be explained by a slowly moving shock front through which the gas in the jet flows. The motion of $\mathrm{C} 2$ has also clearly slowed down since the observations of LJ. Actually, the component already had a lower speed in the UV-observations of Hollis et al. (1997a), assuming that the positions of the components at these two frequencies can be compared.

The binary motion can also have a small effect on the inner jet behaviour. If the accretion disk or the orbit precesses, this should be observed as a transverse component of the proper motion of the inner jet.

We notice that the morphology of the first arcsec of the jet in both directions has remained quite similar from 1991 to 1999. This leads us to consider a rather fixed inner jet, which ends with standing or slowly moving shocks at $\sim 1^{\prime \prime}$ from the core. This part could hide a helical structure, as the configuration of $\mathrm{Clc}, \mathrm{Clb}$ and $\mathrm{Cla}$ (see Fig. 3a) indicates. It resembles the simulated jet of Ouyed et al. (2003), which, however, is at a much smaller scale but, as they suggest, it probably gives some hints for the larger scales, too.

The counter-jet component $\mathrm{A}^{\prime}$ has been removed from most of the proper motion studies because it appeared stationary. In the first attempt we encountered a problem: $\mathrm{A}^{\prime}$ seemed to move towards the core. But as was mentioned above this component is actually in two pieces and in 1992 the more distant end ( $\left.\mathrm{A}^{\prime} \mathrm{a}\right)$ was much brighter (4.0 mJy vs. $0.9 \mathrm{mJy}$ ) and in 1999 the end closer to the core $\left(\mathrm{A}^{\prime} \mathrm{b}\right)$ was a little brighter (3.1 mJy vs.
$2.4 \mathrm{mJy}$ ). Comparing the positions of $\mathrm{A}^{\prime} \mathrm{a}$ and $\mathrm{A}^{\prime} \mathrm{b}$ separately, we found that they both are moving forward, but very slowly. Therefore, we presume that $\mathrm{A}^{\prime}$ is behaving in the same manner as $\mathrm{C} 2$. There are some traces of a jet in front of $\mathrm{A}^{\prime} \mathrm{a}$, too. We also notice the elongation of the core to the SW (Fig. 2), seen in the UV (Hollis et al. 1997a) and at $43 \mathrm{GHz}$ (Hollis et al. 1997c) as well.

If the slowly moving shock scenario is accepted, the gas to the outer jet flows through the shock in the following way: the accreting gas forms the jet close to the core, gets accelerated to a super-Alfvénic jet speed, until it is terminated at shocks C2 and $\mathrm{A}^{\prime}$. At the periastron the flow through the system is enhanced and the core emits a dense gas cloud or a plasmoid (see next section). The dense cloud is accelerated near the base of the jet to a fraction of the speed of the background jet. (Gas can also go past the cloud from the sides.) When the component has passed the shock, it no longer feels the push from the ambient jet and travels on a ballistic trajectory.

\subsubsection{The formation of a jet component}

Accretion on to a compact source (e.g. to the hot companion in R Aqr) and subsequent jet ejection can happen when the accreting star is rapidly rotating and thus creating a strong magnetic field, or when the star possesses a strong field because of its compactness. The rotation period of the hot companion in $\mathrm{R}$ Aqr is difficult to derive because it cannot be observed directly. In the literature, the only rotation period obtained is that of Hollis \& Koupelis (2000), who have derived a rotation period of $\sim 18 \mathrm{yr}$ from their Lorentz force-driven parcel model. Because of the lack of observationally derived parameters, we reject the possibility of a rapidly rotating star as the primary driver mechanism.

The second alternative for the jet driver is the magnetic field of the compact star. For isolated magnetic white dwarfs the 


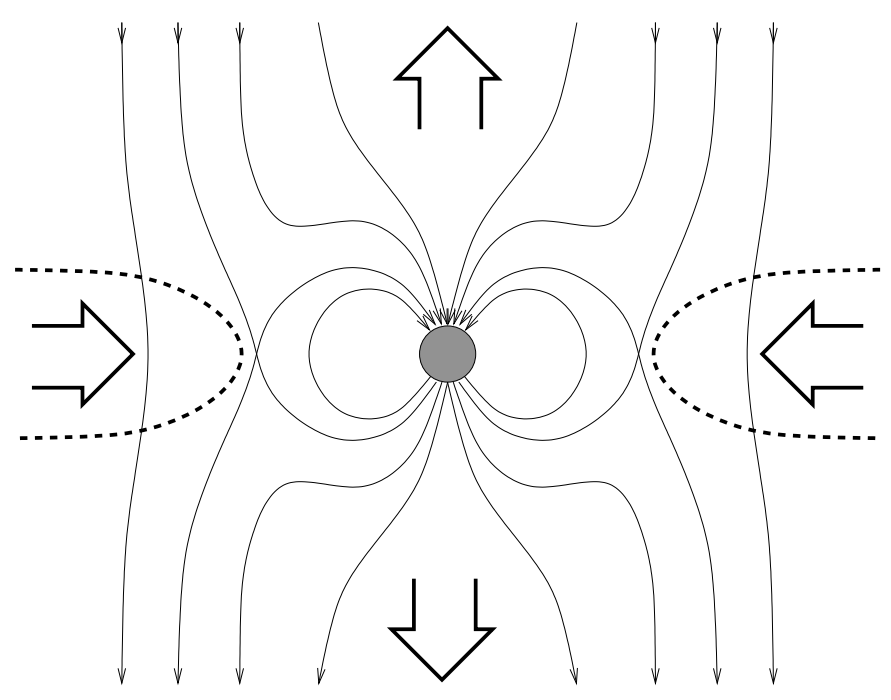

Fig. 4. The reconnection driven jet model of Hirose et al. (1997). The stellar magnetosphere truncates the accretion disk forming a magnetically neutral ring around the star. The jet forms as a result of a reconnection outflow from this X-line. The disk magnetic field is assumed to be parallel to the stellar dipole moment.

surface magnetic field strengths vary between $3 \times 10^{4}-10^{9} \mathrm{G}$ and for magnetic cataclysmic variables (close binaries) between $10^{7}-3 \times 10^{8} \mathrm{G}$ (Wickramasinghe \& Ferrario 2000). Due to the assumed large separation, the companion in R Aqr can be classified as an isolated white dwarf. A model-based estimation comes from Hollis \& Koupelis (2000), who inferred the following surface magnetic field for the hot companion: $B_{*}=\left(9 \times 10^{7}-4.6 \times 10^{9}\right) \mathrm{G}$, if it is a subdwarf of radius $7 \times 10^{9} \mathrm{~cm}$; and $B_{*}=\left(4.4 \times 10^{9}-2.3 \times 10^{11}\right) \mathrm{G}$, if it is a white dwarf of radius of $10^{9} \mathrm{~cm}$. Their model does not take into account any effect due to an accretion disk. The radii used, $7 \times 10^{9} \mathrm{~cm}\left(\approx 0.1 R_{\odot}\right)$ and $10^{9} \mathrm{~cm}$, are based on the studies of Burgarella, Vogel \& Paresce (1992) and Kafatos et al. (1986), respectively.

Another problematic feature is the high escape velocity expected from the surface of a compact star; escape velocities from a $1 M_{\odot}$ white dwarf of $10^{9} \mathrm{~cm}$ are $\sim 5000 \mathrm{~km} \mathrm{~s}^{-1}$ and from a subdwarf of $7 \times 10^{9} \mathrm{~cm} \sim 2000 \mathrm{~km} \mathrm{~s}^{-1}$. Such high velocities have not been observed in R Aqr and it has therefore been assumed that the jet originates far from the hot companionaccretion disk system (Hollis \& Koupelis 2000).

Despite the uncertainty of observational parameters, we test the reconnection-driven model of Hirose et al. (1997) to derive estimations for the starting distance of the jet and the corresponding escape velocity. Hirose et al. have made simulations where no stellar rotation was included. In the model, the stellar dipole moment is parallel to the direction of the magnetic field in the disk (see Fig. 4). The stellar magnetosphere truncates the accretion disk, forming a magnetically neutral ring to the interface between the disk and the magnetosphere at a distance of $r_{\mathrm{m}}$, the truncation radius,

$$
\frac{r_{\mathrm{m}}}{R_{*}} \sim 0.5\left(\frac{B_{*}^{4} R_{*}^{5}}{G M_{*} \dot{M}_{\mathrm{acc}}^{2}}\right)^{1 / 7}
$$

Table 3. Truncation radii in the Hirose et al. model and escape velocities from the distance of the truncation radius for different stellar types (from Hollis \& Koupelis 2000).

\begin{tabular}{cccc}
\hline \hline Radius & Surface magnetic & Truncation & Escape vel. \\
$R_{*}(\mathrm{~cm})$ & field $B_{*}(\mathrm{G})$ & radius $r_{\mathrm{m}}\left(R_{*}\right)$ & $v\left(\mathrm{~km} \mathrm{~s}^{-1}\right)$ \\
$(1)$ & $(2)$ & $(3)$ & $(4)$ \\
\hline $7 \times 10^{9}$ & $9 \times 10^{7}-4.6 \times 10^{9}$ & $287-2718$ & $115-37$ \\
$10^{9}$ & $4.4 \times 10^{9}-2.3 \times 10^{11}$ & $660-6331$ & $201-65$ \\
\hline
\end{tabular}

where $G=6.67 \times 10^{-8} \mathrm{~cm}^{2} \mathrm{~g}^{-1} \mathrm{~s}^{-2}, B_{*}$ is the surface magnetic field, $R_{*}$ the radius and $M_{*}$ the mass of the accreting star. $\dot{M}_{\text {acc }}$ is the accretion rate. The simulation of Hirose et al. shows how the accreting disk mass presses the magnetic field of the disk onto the magnetosphere of the star driving a magnetic reconnection between the magnetospheric field and the disk magnetic field. The angular velocity of the magnetosphere equals the velocity of the Keplerian disk at the corotation radius; for a $1 M_{\odot}$ star having a rotation time of $18 \mathrm{yr}$ the corotation radius $r_{\mathrm{c}}=6.9 \mathrm{AU}$. For the reason that this is much larger than the truncation radius (see calculations below), the star can be considered as non-rotating.

We adopt the above-listed stellar radii for a white dwarf and for a sub-dwarf for our calculations. The accretion rate is estimated as follows: Hollis et al. (1985) have obtained a steady state mass loss of $\sim 2.7 \times 10^{-7} M_{\odot}$ for the LPV. Based on this, Burgarella et al. (1992) derived the accretion rate to the disk of the hot companion to be $\dot{M}_{\text {acc }} \sim 10^{-8} M_{\odot} \mathrm{yr}^{-1}$ assuming that no more than $10 \%$ of the Mira wind can end up in the accretion process. Estimates of the calculated truncation radii (the size of the magnetosphere) of the R Aqr hot companion are shown in Table 3 . We have also calculated the escape velocities to see if the model is reasonable.

The values for the truncation radii with different magnetic field values are quite reasonable: the extrema correspond to $0.13 \mathrm{AU}$ and 1.27 $\mathrm{AU}$ in the case of a subdwarf, and $0.044 \mathrm{AU}$ and $0.42 \mathrm{AU}$ in the case of a white dwarf. These are clearly smaller than the value of the corotation radius, 6.9 AU. The escape velocities at these distances nicely correspond to the suggested typical ejection velocities (e.g. $50 \mathrm{~km} \mathrm{~s}^{-1}$ in Michalitsianos et al. 1988) and the observed velocities of the components.

We point out that the model of Hirose et al. is consistent with the ejection of components (plasma clouds) at periastron: because of the enhanced accretion and mass conservation, more gas flows through the system and enters the jet.

If the magnetic field in the accretion disk is antiparallel to the direction of the stellar dipole moment, reconnection cannot open the field lines of the stellar magnetosphere, or drive a continuous jet along the dipole axis. In this case, however, enhanced accretion during the periastron, if strong enough, could perhaps cause the ejection of plasmoids from the stellar magnetosphere. Increased external ram pressure from the accretion disk first makes the magnetosphere shrink at the equator. Then, as depicted in Fig. 5, a plasmoid can be ejected as a result of reconnection of the dipolar field lines above one of the poles. 


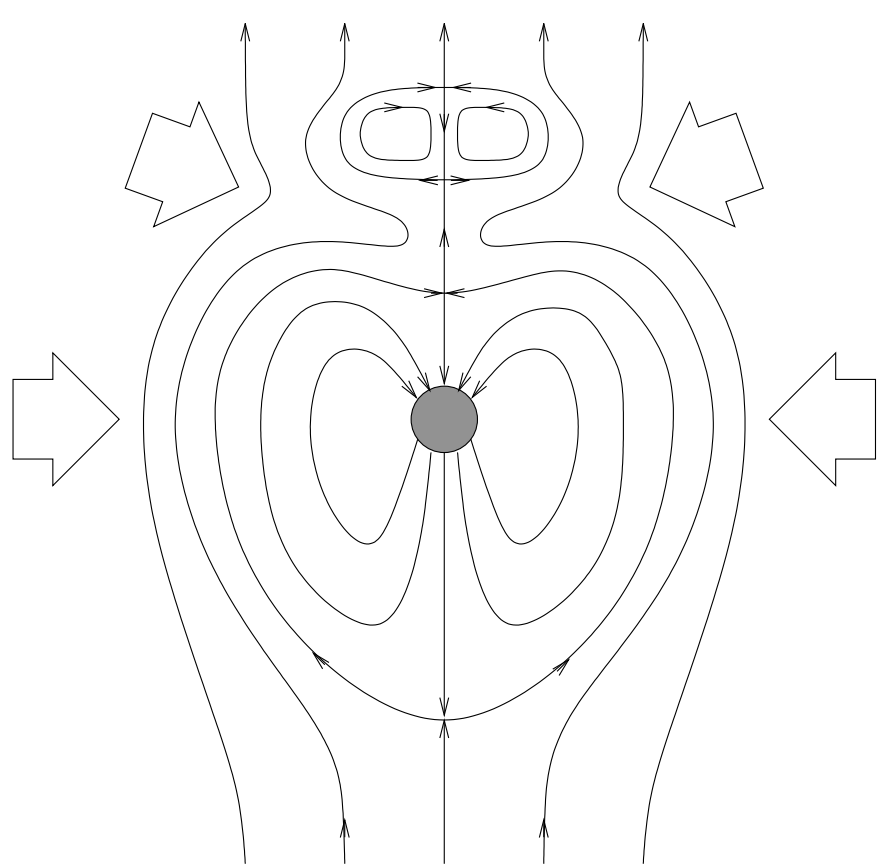

Fig. 5. Ejection of a plasmoid from the stellar magnetosphere. Enhanced accretion during the periastron passage pushes the disk toward the symmetry axis until the dipole field lines reconnect and the plasmoid is ejected from the magnetosphere. The disk magnetic field is assumed to be antiparallel to the stellar dipole moment.

The plasmoid is topologically identical to the mother magnetosphere, and it may propagate along the main dipole axis and be observed as a component on a ballistic track, as long as the ejection occurs far enough from the star. It is unclear, however, whether this model could predict the existence of a collimated continuous jet from the central engine at least if the star is not rotating.

\subsection{The outer jet: Ballistic trajectory and bending}

The distance of A has increased rapidly and the component seems to have experienced an increase in acceleration. Hollis et al. (1997a) measured UV-velocities for A (marked as NE2 in UV) in two terms between 1991.81 and 1993.79. Already these were higher than the radio velocities measured by LJ slightly before. This difference appeared both in radial and transverse motion and the component has been modelled as a lateral shock by Hollis et al. (1997b). Based on the estimated ejection times, LJ assumed that $\mathrm{B}$ and $\mathrm{D}$ had been one single component until they broke apart. We see the same occurring in A. This component was noticed to be elongated in $\mathrm{LJ}$, in 1992.83 we see it coming apart and in 1999.78 it is well separated.

Components B and D are not clearly detected in either observation epoch. Weak features (maximum brightness $0.1 \mathrm{mJy}$ ) at the estimated positions of components $\mathrm{B}$ and $\mathrm{D}$ are detected in 1999.78 (Fig. 1) and their positions are included as a test. It is probable that these components are fading away as was predicted by Burgarella \& Paresce (1991), or cannot be detected in the $\mathrm{A}$ - or $\mathrm{BnA}$-configuration with the frequencies used.

We extrapolated backwards the (radio) breakup time for the component $\mathrm{A}$ and $\mathrm{B}+\mathrm{D}$ assuming that the disrupted components moved with a constant velocity, which should be a valid assumption over a short timescale with low acceleration. For component A, this method yields an epoch 1989.3 for the interception of the separate sub-components, which seems very reasonable considering that $\mathrm{LJ}$ noticed A being unbroken but elongated in 1991.63. In the same way, the disruption epoch of $\mathrm{B}+\mathrm{D}$ would be 1950.1 . Interestingly, both extrapolated disruptions happen at the distance of $\sim 3$ '. 2 , which is near the bend of the jet. Moreover, Kellogg et al. (2001) detected their brightest X-ray component in front of A (or A2), 7'.5 from the core. This component was supposed to be shock-heated and formed less than $\sim 15$ years ago, which is consistent with our disruption time estimate.

According to the studies of Wang et al. (2000), a weak jet can be destroyed by a massive cloud and a dense jet may be bent. In case it is a plasmoid, the disruption of A could be explained by a collision from the side with a more massive cloud; the morphology of A in Hollis et al. (1997a) also seems to support this idea. As a consequence of the collision, the inner magnetic field of the blob would flatten, then reconnect in the middle and finally break up, just as in case of the original ejection. A collision would also explain the bending seen at A.

Another possibility is that a reverse shock is propagating in component A (see Hartigan \& Raymond 1993). In this case, a collision with a cloud in front of A could cause the shock and subsequent disruption. A moving shock with a velocity of 90-100 $\mathrm{km} \mathrm{s}^{-1}$ was suggested to heat B and D in the recent past (Burgarella \& Paresce 1991). Note that the low velocity does not have to be in conflict with the shock hypothesis, if the components are dense enough to make their internal Alvén speed small. It is possible that the same mechanism, a shock and a reverse shock, has heated and disrupted B and D and now A. It is also possible that the shock started somewhere else in the jet (such as the core) and catches up the components and interacts with them later. In this case, one could assume that the shock is weak (and thus invisible) while propagating along the jet until it meets the plasma cloud with the slow signal speed, where it can be strengthened because of the increase in its Mach number.

We have plotted (see Fig. 6) the position of components in three different epochs covering $\sim 16$ years period. The angle between the outermost jet paths of the NE jet is about $11^{\circ}$ measured at $\sim 4^{\prime \prime}$, similar to the result of Hollis et al. (1997b). If the lateral motion does not represent the impact from the proper motion of the system, but the binary motion or precession of the disk, one would expect the path to turn back or the lateral motion to slow down in a time period of half the originator's period.

\section{Summary}

We have observed the jet of R Aqr at high resolution with the VLA. We could not detect the expected new jet component. Periodic ejection of matter is still a possible scenario. Either 


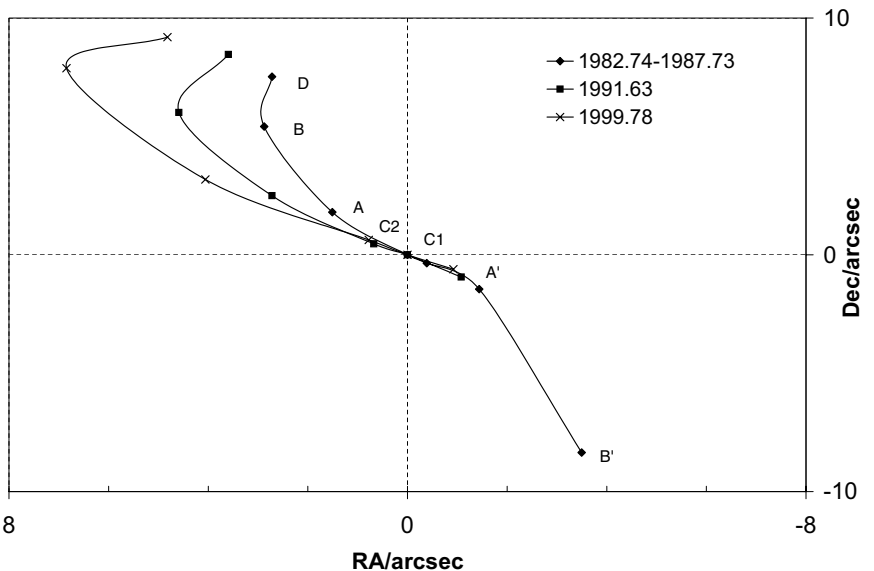

Fig. 6. The positions of components relative to core between 1982.74 and 1999.78. We have combined the positions of the five-years period during the 80's. The positions of these epochs are taken from Kafatos et al. (1983) (A and B), Hollis et al. (1986) (C1 and C2), Kafatos et al. (1989) $\left(\mathrm{A}^{\prime}\right.$ and $\left.\mathrm{B}^{\prime}\right)$ and $\mathrm{LJ}(\mathrm{D})$.

$\mathrm{C} 1 \mathrm{a}+\mathrm{b}$ is a new component, which implies a little shift in time interval between ejections, or the timing based either on the models or the periastron passage is wrong. We have discussed, based on the known restrictions, how the components can be ejected and accelerated far from the hot companion/accretion disk system.

Based on the proper motion analysis, we find that the base of the jet is moving very slowly. The first $1^{\prime \prime}$ in both directions consists of a fixed, slowly-moving part of the jet. This part is controlled by the magnetic field of the hot companion/accretion disk system and can be considered as an acceleration field. In this field the gas or the components are accelerated to superAlfvénic speed. Shocks are created at the location of C2 and $\mathrm{A}^{\prime}$ at a distance of $\sim 1^{\prime \prime}$ from the core. After the shock front the gas encounters a sub-Alfvénic medium and travels on a ballistic orbit. $\mathrm{A}^{\prime}$ has been excluded from the previous models because it seemed to move towards the core. This discrepancy is explained by the breakup into two separate components.

In the outer part of the jet we see the NE component $\mathrm{A}$ breaking apart in a similar manner to what seems to have happened to B and D earlier (see LJ). A possible explanation for the disruption is a collision with a cloud, causing either a reconnection in the component's internal magnetic field lines, or a reverse shock.

Acknowledgements. We thank NRAO AOC staff for their hospitality and help. This work was partially supported (K.M. and H.L.) by grant number 44011 from Academy of Finland. K.M. thanks The Finnish Graduate School in Astronomy and Space Physics for financial support. D.R.H.J. thanks Charterhouse for its support of this work.

\section{References}

Burgarella, D., \& Paresce, F. 1991, ApJ, 370, 590

Burgarella, D., Vogel, M., \& Paresce, F. 1992, A\&A, 262, 83

Dougherty, S. M., Bode, M. F., Lloyd, H. M., Davis, R. J., \& Eyres, S. P. 1995, MNRAS, 272, 843

Hartigan, P., \& Raymond, J. 1993, ApJ, 409, 705

Hinkle, K. H., Wilson, T. D., Scharlach, W. W. G., \& Fekel, F. C. 1989, AJ, 98, 1820

Hirose, S., Uchida, Y., Shibata, K., \& Matsumoto, R. 1997, PASJ, 49, 193

Hollis, J. M., Bertram, R., Wagner, R. M., \& Lampland, C. O. 1999, ApJ, 514, 895

Hollis, J. M., Kafatos, M., Michalitsianos, A. G., \& McAlister, H. A. 1985, ApJ, 289, 765

Hollis, J. M., Michalitsianos, A. G., Kafatos, M., Wright, M. C. H., \& Welch, W. J. 1986, ApJ, 309, 53

Hollis, J. M., Kafatos, M., Michalitsianos, A. G., Oliversen, R. J., \& Yusef-Zadeh, F. 1987, ApJ, 321, L55

Hollis, J. M., \& Koupelis, T. 2000, ApJ, 528, 418

Hollis, J. M., Lyon, R. G., Dorband, J. E., \& Feibelman, W. A. 1997a, ApJ, 475, 231

Hollis, J. M., Pedelty, J. A., \& Kafatos, M. 1997b, ApJ, 490, 302

Hollis, J. M., Pedelty, J. A., \& Lyon, R. G. 1997c, ApJ, 482, L85

Ilovaisky, S. A., \& Spinrad, H. 1966, PASP, 78, 527

Kafatos, M., \& Michalitsianos, A. G. 1982, Nature, 298, 540

Kafatos, M., Hollis, J. M., \& Michalitsianos, A. G. 1983, ApJ, 267, L103

Kafatos, M., Michalitsianos, A. G., \& Hollis, J. M. 1986, ApJS, 62, 853

Kafatos, M., Hollis, J. M., Yusef-Zadeh, F., Michalitsianos, A. G., \& Elitzur, M. 1989, ApJ, 346, 991

Kellogg, E., Pedelty, J. A., \& Lyon, R. G. 2001, ApJ, 563, L151

Kharchenko, N., Kilpio, E., Malkov, O., \& Schilbach, E. 2002, A\&A, 384,925

Lang, K. 1992, in Astrophysical data: Planets and stars (New York: Springer-Verlag, Inc.), 693

Lehto, H. J., \& Johnson, D. R. H. 1992, Nature, 355, 705 (LJ)

Meier, S., \& Kafatos, M. 1995, ApJ, 451, 359

Merrill, P. W. 1950, ApJ, 112, 514

Michalitsianos, A. G., \& Kafatos, M. 1982, ApJ, 262, L47

Michalitsianos, A. G., Oliversen, R. J., Hollis, J. M., et al. 1988, AJ, 95, 1478

Ouyed, R., Clarke, D. A., \& Pudritz, R. E. 2003, ApJ, 582, 292

Paresce, F., \& Hack, W. 1994, A\&A, 287, 154

Solf, J., \& Ulrich, H. 1985, A\&A, 148, 274

Solf, J. 1992, A\&A, 257, 228

Sopka, R. J., Herbig, G., Kafatos, M., \& Michalitsianos, A. G. 1982, ApJ, 258, L35

Teräsranta, H., Tornikoski, M., Mujunen, A., et al. 1998, A\&AS, 132, 305

Wang, Z., Wiita, P. J., \& Hooda, J. S. 2000, ApJ, 534, 201

Van Leeuwen, F., Feast, M. W., Whitelock, P. A., \& Yudin, B. 1997, MNRAS, 287, 955

Wickramasinghe, D. T., \& Ferrario, L. 2000, PASP, 112, 873

Willson, L. A., Garnavich, P., \& Mattei, J. A. 1981, IBVS, 1961, 1 